\title{
Reflets
}

Revue d'intervention sociale et communautaire

\section{Reflets a 20 ans! Si cette revue n'existait pas, il faudrait l'inventer}

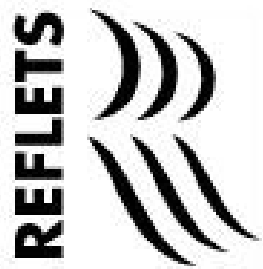

\section{Gabriel Marin et Nérée St-Amand}

Volume 21, numéro 2, automne 2015

$20^{\mathrm{e}}$ anniversaire... déjà !

URI : https://id.erudit.org/iderudit/1035434ar

DOI : https://doi.org/10.7202/1035434ar

Aller au sommaire du numéro

Éditeur(s)

Reflets, Revue d'intervention sociale et communautaire

ISSN

1203-4576 (imprimé)

1712-8498 (numérique)

Découvrir la revue

Citer cet article

Marin, G. \& St-Amand, N. (2015). Reflets a 20 ans! Si cette revue n'existait pas, il faudrait l'inventer. Reflets, 21(2), 71-111. https://doi.org/10.7202/1035434ar
Résumé de l'article

Cet article examine les vingt ans d'existence de la revue scientifique et professionnelle Reflets, créée en 1995. Tout en regardant son impact, ses défis et ses perspectives, l'article rend hommage aux pratiques de créativité et de détermination de la communauté franco-ontarienne et aux sources de soutien de la revue dans un contexte de défis structurels importants.
Tous droits réservés (C) Reflets, Revue d'intervention sociale et communautaire, 2015 document est protégé par la loi sur le droit d'auteur. L'utilisation des services d'Érudit (y compris la reproduction) est assujettie à sa politique d'utilisation que vous pouvez consulter en ligne. 


\section{Reflets a 20 ans! Si cette revue n'existait pas, il faudrait l'inventer}

Franco-Ontariens, Canadiens-Français, francophones, francophones hors Québec, Ontarois. Ce sont autant de termes qui témoignent du dilemme identitaire qui, aujourd'hui encore, caractérise si bien l'Ontario français (Bock, 2001, p. 9).

Gabriel Marin, Ph. D.

Étudiant à la maîtrise, École de service social, Université d'Ottawa

Nérée St-Amand, Ph. D.

Professeur titulaire, École de service social, Université d'Ottawa

\section{Résumé}

Cet article examine les vingt ans d'existence de la revue scientifique et professionnelle Reflets, créée en 1995. Tout en regardant son impact, ses défis et ses perspectives, l'article rend hommage aux pratiques de créativité et de détermination de la communauté franco-ontarienne et aux sources de soutien de la revue dans un contexte de défis structurels importants.

Mots clés : minorités franco-ontariennes, travail social, histoire, politiques sociales, intervention sociale, intervention en contexte minoritaire.

\section{Abstract}

This article examines the 20 years of existence of the professional journal Reflets, launched in 1995. While looking at the journal's impact, challenges and perspectives, it pays tribute to the empowerment practices of the Franco-Ontarian communities 
who were able to create and develop this journal, in spite of few resources and many structural challenges.

Key words: minorities, Franco-Ontarian, social work, history, social policy, social intervention, minority context.

\section{Introduction}

"Dès le départ, ce comité a privilégié une approche ancrée sur le terrain en établissant un partenariat entre les milieux universitaires et communautaires, comblant ainsi une lacune bien évidente dans un contexte où à l'époque "les intervenantes et les intervenants qui ceuvrent auprès de la communauté francophone dans le domaine des pratiques sociales et communautaires n'ont aucun outil de communication qui leur soit propre »...»
Reflets a 20 ans! Ces mots font écho à la célèbre chanson de Moustaki dont certaines des paroles — " votre fille a vingt ans, que le temps passe vite... »- peuvent se fredonner conjointement avec les sentiments qui entourent les vingt ans de la revue : le temps qui passe, les gens qui restent, qui vieillissent et qui changent, etc., tout en faisant un clin d'œil à l'histoire qu'il ne faut surtout pas oublier.

Reflets a 20 ans! C'est en 1995 en effet que la revue est née de l'initiative d'une vingtaine de personnes enthousiastes représentant divers milieux franco-ontariens, aussi bien universitaires que sociocommunautaires. Huit personnes, Richard Carrière (Sudbury), Richard Martel (Toronto), Hélène Gagné (Toronto), Michèle Kérisit (Ottawa), Chris Sassa (Toronto), Céline Simard (Sturgeon Falls), Nérée St-Amand (Ottawa) et Madeleine Dubois (Ottawa), ont fait partie du comité de fondation de la revue. Dès le départ, ce comité a privilégié une approche ancrée sur le terrain en établissant un partenariat entre les milieux universitaires et communautaires, comblant ainsi une lacune bien évidente dans un contexte où à l'époque " les intervenantes et les intervenants qui œuvrent auprès de la communauté francophone dans le domaine des pratiques sociales et communautaires n'ont aucun outil de communication qui leur soit propre " (Carrière, 1995, p. xi). Par ailleurs, Reflets se proposait d'agir comme un portevoix des besoins de la communauté francophone minoritaire dans sa quête identitaire, en lien avec son passé de luttes historiques et l'avenir incertain qui se dessine sous le gouvernement conservateur de l'époque. En un sens, l'Ontario français d'alors était constitué — et il l'est toujours — d'ilots minoritaires 
dispersés dans une mer anglophone, formés de gens qui tissaient à travers le temps et l'histoire une identité particulière autour de liens culturels, politiques, économiques et, il va sans dire, sociaux-communautaires. L'histoire démontre que la population francophone de l'Ontario n'avait pas nécessairement un pouvoir politique officiellement reconnu, mais cela n'a pas empêché les luttes héroïques menées pour atteindre une complétude institutionnelle — l'expression est de Breton (1964) —, entre autres, sur le plan scolaire et sur celui de l'offre de services en français, particulièrement dans les domaines de la santé et des services sociaux. À travers de grandes revendications, souvent en utilisant le concept des droits, de nombreux dossiers ont évolué et le peuple franco-ontarien s'est acquis une fierté bien justifiée. C'est dans un tel contexte que Reflets : revue ontaroise d'intervention sociale et communautaire voit le jour, donnant ainsi une autre voix à une communauté déjà aguerrie face à l'adversité, qu'elle soit culturelle, linguistique, politique ou sociale. Soulignons également que cette interaction avec l'« autre " ne touche pas uniquement la population anglophone de l'Ontario ou du Canada, mais aussi celle du Québec, là où le contexte sociopolitique relègue souvent au second plan les intérêts immédiats des Franco-Ontariennes et des Franco-Ontariens, tout comme ceux du peuple acadien et d'autres groupes minoritaires canadiens.

Ainsi, dans les années 1990, diverses particularités d'ordre historico-politique amèneront les personnes intéressées à créer une revue d'intervention visant à reconnaître et à mettre en valeur cette condition franco-minoritaire de l'Ontario français. Il n'était guère étonnant de constater dès la première publication de la revue une quête d'affirmation de cette identité en lien avec un idéal d'émancipation politique.

La communauté est présentement confrontée à des changements sociaux, économiques et politiques de taille qui amènent une remise en question de ses modes de définition comme collectivité. Depuis les débuts de leur histoire, les Franco-Ontariennes et Franco-Ontariens n'ont pas été des victimes passives, mais des 
"Ce statut particulier d'un peuple apatride à l'extérieur de la géographie identitaire du Québec témoigne de ce qu'Arendt qualifie de "stateless" qui se caractérise par l'absence d'un pouvoir politique institutionnalisé pour le représenter..." acteurs engagés dans la transformation de leurs communautés. C'est en reconstituant cette histoire collective qu'ils seront en mesure de faire face aux transformations actuelles en leur donnant une nouvelle finalité. (Welch, 1995, p. 21)

Ce positionnement face à l'identité et vis-à-vis de la différence fait des francophones vivant en milieu minoritaire au Canada des témoins engagés dans une histoire où plusieurs lectures de l'exclusion sociale (classe, race, genre) se superposent et les affectent en façonnant leur conscience à la fois orientée sur le "soi " (conscience de leur communauté) et sur l'" autre ", conscience d'un social complexe en raison de son contexte historico-politique. Ce "vivre-ensemble " à côté de l'" autre " d'une minorité qui parle une langue dont la reconnaissance n'est toujours pas officialisée place d'ailleurs la communauté francoontarienne à l'avant-garde des lieux de passages entre cultures (Benjamin, 1939; [2003]). Ce statut particulier d'un peuple apatride à l'extérieur de la géographie identitaire du Québec témoigne de ce qu'Arendt qualifie de "stateless " qui se caractérise par l'absence d'un pouvoir politique institutionnalisé pour le représenter : «La perte de patrie et de statut politique revient à être expulsé de l'humanité tout entière." (Arendt, 1955; [2002a], p. 599) Trois documents (The Public School Act [1885],le Règlement du ministère de l'Éducation d'Ontario [1890] et surtout le Règlement XVII ou la Circulaire 17 [1912]) rappellent que le prix à payer pour cette lutte identitaire s'observait déjà au début du $20^{\mathrm{e}}$ siècle en rapport avec la question de l'éducation en français en Ontario. Le Règlement XVII est devenu depuis l'un des grands moteurs et symboles de la survie et de la fierté identitaire des communautés franco-ontariennes.

L'adoption du tristement célèbre Règlement XVII par le gouvernement ontarien en 1912 représente la plus importante menace au développement des écoles franco-ontariennes. Pendant quinze ans, la crise scolaire provoquée 
par le Règlement XVII est ponctuée de plusieurs luttes acerbes. Mais cette crise permet néanmoins l'émergence d'une plus grande cohésion et une meilleure convergence des forces en faveur de l'éducation de langue française en Ontario. Créée en 1910, l'Association canadienne-française d'éducation d'Ontario (ACFEO) est la porte-parole incontestée de la communauté franco-ontarienne. (CRCCF, 2004)

L'Annexe 1 rappelle quelques aspects originaux de cette revendication :les stratégies et discours politiques pour les hommes et la guerre des rouleaux à pâte et des épingles à chapeaux pour les femmes. Près d'un siècle plus tard, nous assisterons à une autre lutte, pour des institutions de santé en français cette fois, illustrée dans le contexte d'une fermeture décrétée de l'Hôpital Montfort. Une autre victoire d'un peuple qui sait s'unir autour de questions d'identité et de droits (voir l'article de Perron Roach dans le présent numéro)! Autour de ces crises, pour ne mentionner que celles-là, un projet de société franco-ontarienne prend vie et les luttes pour une éducation et un réseau de santé et de services sociaux en français contribuent à l'affirmation d'un positionnement politique des francophones d'ici et même d'ailleurs au Canada. D'un point de vue politique, ces luttes ont créé un contrepoids au pouvoir et favorisé un esprit de solidarité, une conscience historique et un sentiment d'appartenance à une province et à un pays dans un contexte où une minorité refuse une oppression historico-politique solidement ancrée ${ }^{1}$.

Comment ce mot Franco-Ontarien consacre-t-il une telle aliénation? En tant que mot composé où la "francité " du citoyen est non seulement troquée dans l'élément " franco ", mais aussi refoulée, écartée, mise en quelque sorte à l'écart, à la remorque d'une majorité ontarienne qui refuse, en fait, de composer vraiment dans la réalité. (Grisé, 1982, p. 87) 
"...deux conceptions politiques s'affrontaient et continuent de le faire : une qui veillait à garder l'élément français à l'ombre, pire au rancart, l'autre où les Canadiennes françaises et les Canadiens français revendiquaient leurs droits, croyant en un Canada bilingue où le français serait à l'égal de l'anglais, d'où le concept de complétude institutionnelle de Breton...»
En réalité, deux conceptions politiques s'affrontaient et continuent de le faire : une qui veillait à garder l'élément français à l'ombre, pire au rancart, l'autre où les Canadiennes françaises et les Canadiens français revendiquaient leurs droits, croyant en un Canada bilingue où le français serait à l'égal de l'anglais, d'où le concept de complétude institutionnelle de Breton (Thériault, 2014). D'ailleurs, devenu un outil de conscientisation pour les communautés minoritaires, ce concept est présenté par Cardinal et Gonzàlez Hidalgo (2012, p. 62) comme « [u]n droit à la complétude institutionnelle [qui] devrait être envisagé pour les minorités comme les francophones hors Québec, à l'instar du droit à l'autodétermination pour les minorités nationales ou du droit à la non-discrimination pour les minorités ethniques".

En 1995, les artisans de Reflets avaient-ils en tête tous les éléments de cette analyse historico-politiques? Le présent article tentera de répondre à cette question en présentant certains accomplissements à inscrire à côté d'autres victoires de ce peuple vivant en contexte minoritaire, mais mu par des convictions fortes et faisant preuve de stratégies de survie toujours en renouvèlement.

\section{L'écriture et affirmation identitaire}

La nécessité d'une revue d'intervention, tant sur le plan idéologique, politique, social que stratégique, se définit d'ores et déjà dans le contexte d'une reconnaissance des conditions particulières existantes. « Nous nous proposons [...] d'identifier et d'explorer les caractéristiques faisant en sorte que l'intervention en Ontario français ne puisse pas reproduire ce qui se passe ailleurs, en raison des particularités historico-socio-politiques en contexte minoritaire ", écrivait Carrière dans le premier numéro (1995, p. xi). L'avenir lui donnera raison. Aujourd'hui, à l'heure du bilan et de la célébration des vingt ans de Reflets, les intérêts liés aux causes structurelles de l'exclusion sociale ressortent clairement de ses thèmes et de ses articles et de son orientation vers l'intervention sociale. 
Dans les prochains paragraphes, nous passons en revue les grands thèmes abordés dans Reflets au fil de ses publications. Nous en étudions les impacts sur différentes formes d'intervention, en nous basant, entre autres, sur un sondage d'opinion mené auprès de personnes qui ont collaboré à la revue. En conclusion, nous proposons quelques pistes qui émergent de cette étude des articles parus pour en dégager des orientations possibles et, nous le souhaitons, quelques leçons d'un historique qui relève de l'exemple à suivre.

\section{Il $\mathrm{y}$ a vingt ans...}

"...on retrouve ce souci de se doter d'instruments de compréhension de sa propre réalité de francophone vivant en milieu minoritaire. C'est dans ce contexte que seront définis la mission et les objectifs de la revue..."
La question qui se posait au départ était la suivante : quelle place cette nouvelle revue pourrait-elle occuper par rapport à l'ensemble des publications du même genre au Canada français? Qu'est-ce qui la distinguerait des autres? S'agirait-il d'une spécificité, d'une organisation axée autour de problématiques précises comme le fait une autre revue de la francophonie minoritaire, la Revue de l'Université de Moncton ${ }^{2}$ ? Se consacrerait-elle comme Nouvelles pratiques sociales à la nouveauté sur le plan des interstices de l'univers social et politique? Reflèterait-elle les préoccupations d'un devoir citoyen (Ricœur, 2000) pour préparer les actrices et acteurs du social à bien comprendre les contradictions d'une société traversée par autant d'inégalités que d'espoirs? Ou encore s'attarderait-elle aux problèmes des gens ordinaires, poussés à l'invention du quotidien, comme le propose Certeau (1980)? Et dans ces questionnements, comment la revue se ferait-elle l'écho d'attentes et d'expériences tout aussi complexes que contradictoires à l'heure d'une mondialisation par le bas (Tarrius, 2002) ou de la dispersion ethnique (Ma Mung, 1999) de mondes à la fois isolés, mais solidaires?

$\mathrm{Au}$-delà des grandes questions philosophiques qui habitaient les personnes attentives à la mouvance sociale, on retrouve ce souci de se doter d'instruments de compréhension de sa propre réalité de francophone vivant en milieu minoritaire. C'est dans ce contexte que seront définis la mission et les objectifs de la revue (voir Annexe 2). 
Dès lors, de nombreuses tâches se sont imposées, entre autres, définir la mission de la revue, identifier les thèmes des premiers numéros, choisir les diverses rubriques, lancer des appels de communications, de même que trouver un titre approprié, du financement et une maison d'édition. Le soutien financier du ministère des Services sociaux et communautaires et du ministère de la Santé ont facilité de beaucoup l'exécution des multiples tâches afférentes à la publication de la revue.

Le lancement de Reflets en 1995 repose sur une demande à la fois sociale et professionnelle. Pour concrétiser sa vision et ses idéaux, la revue adopte un canevas constitué d'un Éditorial, d'une Entrevue, d'un Dossier regroupant autour d'une thématique divers articles arbitrés, de même que d'autres rubriques aux noms évocateurs, à savoir, Des pratiques à notre image, Aux quatre coins de la province, Aux études et Lu pour vous (Dubois, et St-Amand, 1995, p. xvii).

Au printemps de 1995, au bout de deux ans de gestation, Reflets présente à la fois son nom, son logo et publie son premier numéro sous le thème Des pratiques socio-communautaires à notre image. Les six articles arbitrés et treize autres textes l'ensemble provenant de sources diverses et épousant des perspectives tout aussi diverses en font un numéro fort apprécié. Le ton est donné, le projet prend son envol! L'engagement et ses défis se conjuguent dès le départ par l'usage du verbe "écrire ", mentionné d'emblée par les deux coresponsables de ce premier numéro. Ainsi, on souhaite dès lors une revue axée sur le changement social par le biais de l'écriture, un outil de sensibilisation, certes, mais aussi de mobilisation politique.

Dans la création de ce premier numéro, le verbe écrire a été conjugué à tous les temps et a donné lieu à un foisonnement social et communautaire nous caractérisant... pratiques tant communautaires qu'académiques et institutionnelles, pratiques des intervenantes et des intervenants, des étudiantes et des étudiants des diverses régions, bref, pratiques des 
"Ce qui est décidé de façon plus ou moins intuitive, c'est la mise en récit d'une "mémoire silencieuse ", ... soit la mémoire d'une communauté fière de son histoire malgré des politiques et interventions étatiques marquées par la discrimination, sinon l'exclusion."

"...la résistance est valorisée dans l'article de Welch tout comme dans de nombreux articles qui suivront; en somme, certaines stratégies originales d'intervention s'affichent d'ores et déjà, à l'image du peuple dont il reflète les aspirations." personnes engagées à de multiples niveaux dans des réflexions sur le sens et l'orientation de l'intervention sociale en Ontario français (Dubois, et St-Amand, 1995, p. xvii).

Ce qui est décidé de façon plus ou moins intuitive, c'est la mise en récit d'une "mémoire silencieuse ", au sens de Ricœur (2000)3, soit la mémoire d'une communauté fière de son histoire malgré des politiques et interventions étatiques marquées par la discrimination, sinon l'exclusion. C'est pourquoi, dès le premier numéro, on cherche à évaluer les enjeux et les ressources qui sont au carrefour d'un passé de luttes et d'un avenir rempli de défis; en effet, comment ne pas rappeler que 1995, c'est aussi l'année où Mike Harris se fait élire en Ontario, se donnant comme agenda d'éliminer tout ce qui se conjugue avec social, État-providence, et pire encore, le fait français :

Mike Harris's first year in government is highly reminiscent of the early Thatcher years in Great Britain. The Harris Tories have espoused a Thatcherite philosophy, a potent neo-conservative brew of neoliberal economics and "authoritarian populist" social policy. They have the same fundamental objective, the redistribution of income from the "worse-off" to the "better-off" sections of society. (Leduc-Browne, 1996, p. 37)

Malgré ces défis de taille, les artisanes et artisans de la première heure brossent un tableau prometteur "des caractéristiques qui font partie de notre histoire et qui se tissent dans les pratiques que nous inventons ou développons» (Dubois, et St-Amand, 1995, p. xviii). Dans ce contexte, ce n'est pas un hasard si le Dossier de ce premier numéro (Vol. 1, № 1, printemps 1995) s'ouvre sur un article de Welch proposant justement un survol historique et se referme sur celui de Molgat et Lemire, un article qui s'intéresse aux jeunes, donc à l'avenir de la communauté franco-ontarienne. Soulignons de plus que la résistance est valorisée dans l'article de Welch tout comme dans de nombreux articles qui suivront; en 
somme, certaines stratégies originales d'intervention s'affichent d'ores et déjà, à l'image du peuple dont il reflète les aspirations.

Ce bref clin d'œil historico-politique permet de bien appréhender certains des défis d'alors dont les codirecteurs font état dans l'Éditorial et dans la présentation de ce premier numéro :

Ce souci de nommer et de faire face aux problèmes exprimés par les gens constitue un fil conducteur sous-tendant les articles de ce numéro. Les problèmes de pauvreté, d'isolement, de chômage et de manque de formation, liés à des questions d'identité et de revendication de droits, constituent autant de défis pour toute personne intervenant en Ontario français. Ces thèmes ressortent déjà au départ. Le désir de venir en aide aux personnes plus marginalisées se dégage de nombreux articles de la rubrique Aux quatre coins de la province. Nous y présentons des services, des programmes et des initiatives francophones dans les domaines de la promotion de la santé, des services sociaux scolaires, de la violence conjugale, de l'agression sexuelle et de la santé mentale. (Dubois, et St-Amand, 1995, p. xix).

Cette évocation du contexte de 1995 permet d'entrevoir le programme et la raison d'être de Reflets. Sa mission se concrétise à travers ces idéaux de départ, non seulement en ce qui a trait aux thèmes choisis, mais aussi par l'approche et le ton d'où se dégage, en quelque sorte, sa " couleur".

Soulignons enfin quelques modifications qu'a connues la revue au fil de ses publications. Connu lors de son lancement en tant que Reflets : revue ontaroise d'intervention sociale et communautaire, son comité de direction a décidé en 2008 d'en modifier le titre.Ainsi, dans le but d'en accroître le rayonnement, la revue porte désormais le titre de Reflets : revue d'intervention sociale et communautaire. 
Au départ, la revue était semestrielle et elle l'est redevenue en 2010, après n'avoir été publiée qu'une fois l'an de 2004 à 2009.Vous feuilletez actuellement le $35^{\mathrm{e}}$ numéro de Reflets : revue d'intervention sociale et communautaire.

\section{Les thèmes de la revue}

"...Reflets $a$ publié un total de 35 numéros totalisant 384 articles ainsi répartis : 145 dans Le Dossier, 148 dans Des pratiques à notre image $e t$ 101 dans Aux quatre coins de la province. »
Écrire est, qu'on le veuille ou non, un verbe politique... l'écriture n'engendre sans doute pas l'identité collective, mais élucide ses lieux d'origine (Paré, 1994, p. 61).

En tenant compte de son édition actuelle, Reflets a publié un total de 35 numéros totalisant 384 articles ainsi répartis : 145 dans Le Dossier, 148 dans Des pratiques à notre image et 101 dans Aux quatre coins de la province. En somme, une contribution importante du domaine académique, appuyée par un nombre imposant d'articles dont les signataires proviennent des milieux de pratique, ce qui témoigne d'un engagement soutenu à publier de la part de la communauté. Rappelons qu'en 2003, le comité éditorial a décidé de fusionner les rubriques Des pratiques à notre image et Aux quatre coins de la province en une seule intitulée Des pratiques à notre image. En plus, chaque numéro comporte une section $L u$ pour vous qui propose diverses recensions de textes pertinents aux thèmes présentés. Enfin, la rubrique $A u x$ études met en valeur les finissantes et les finissants de l'Université d'Ottawa et de l'Université Laurentienne en présentant un résumé de leurs mémoires de maîtrise en service social.

Les thèmes et sujets abordés au cours de vingt années de publication méritent quelques commentaires et soulèvent des questions supplémentaires qui se dégagent de ce rapide coup d'œil des contenus présentés. Des titres des trente-cinq numéros déjà parus, certains thèmes ressortent clairement d'au moins la moitié d'entre eux : les termes "pratique " (9 occurrences) et "intervention " (9 occurrences également) tiennent la tête d'affiche, confirmant ainsi une tendance qui se dégage des 384 articles publiés. En voici quelques exemples : Travail, jeunesse et 
intervention (Vol. 14, $\left.\mathrm{N}^{\circ} 1,2008\right)$, L'intervention communautaire chez les francophones minoritaires : des pratiques à découvrir (Vol. 18, $\mathrm{N}^{\circ}$ 1, printemps 2012), Approches d'intervention : définir et renouveler nos pratiques (Vol. 6, № 1 , printemps 2000), Approches structurelles et intervention sociale (Vol. 19, № 1, printemps 2013). Par ailleurs, le terme "intervention " rallie un nombre important de préoccupations, surtout lorsqu'il est joint à d'autres termes comme " social ", « approches ", « jeunesse ", « féminisme ", " vieillissement " ou «travail ». En somme, les thèmes abordés donnent un aperçu des horizons que la revue a explorés depuis son lancement. Et lorsque "intervention » ou "pratique " côtoient des termes tels que "service social ", " professionnelle ou professionnel de l'aide ", " enseignement » ou "formation ", nous disposons d'un portrait de quelques-unes des richesses et complexités des perspectives présentées, dont :

- la psychologisation de l'intervention sociale;

- le contexte minoritaire;

- la dimension interculturelle;

- la paternité;

- la spiritualité;

- la souffrance psychique et morale au travail;

- le développement économique communautaire;

- la formation pratique.

Ces thèmes confirment et concrétisent la mission initiale de la revue d'intervention sociale et communautaire. Sur le plan des choix thématiques, un autre terme clé ressort, soit celui de " minorité ", avec ses dérivés " minoritaire » et " minorisation ». Dès 1998, un numéro complet y est consacré, Intervention en contexte minoritaire (Vol. 4, № 1, printemps 1998). Ce thème regroupe tout un réseau de signification autour d'autres termes ou locutions : " intervention ", « francophone ", " Ontario français ", "santé ", "services sociaux ", « accès aux services " ou " développement de services en français ». Huit numéros sont clairement consacrés à la question minoritaire et la grande majorité des articles arbitrés aborde cette dimension. 
"Nous notons qu'une réflexion critique est présente de façon plus ou moins explicite dans la très grande majorité des thèmes abordés..."
Par ailleurs, le terme " approche " constitue un autre concept autour duquel gravitent plusieurs préoccupations, par exemple, Approches d'intervention : définir et renouveler nos pratiques (Vol. 6, $\mathrm{N}^{\circ}$ 1, printemps 2000) et Approches structurelles et intervention sociale (Vol. 19, No 1, printemps 2013).

Nous notons qu'une réflexion critique est présente de façon plus ou moins explicite dans la très grande majorité des thèmes abordés : Le genre en contexte : pratiques sociales et représentations (Vol. 9, № 1, printemps 2003), La violence dans tous ses états (Vol. $\left.13, N^{\circ} 1,2007\right)$, Les inégalités sociales de santé chez les communautés francophones en situation minoritaire (CFSM) au Canada (Vol. 18, N ${ }^{\circ}$ 2, automne 2012), Problèmes sociaux en Ontario français (Vol. 6, $\mathrm{N}^{\circ} 2$, automne 2000), Santé mentale et défis de l'année 2001 (Vol. 7, N 1, printemps 2001), Violences faites aux femmes et contextes minoritaires (Vol. 21, No 1, printemps 2015). D'ailleurs, reflétant les nombreux enjeux dans le domaine de la santé, nous constatons un important noyau d'articles abordant l'intervention en contexte de santé. Sept thèmes y sont consacrés et ce concept se situe au carrefour des problèmes d'accès, des disciplines, des communautés, de la formation et de la francophonie en contexte minoritaire. En plus, la santé est présentée en liens avec d'autres thèmes comme l'incapacité, le vieillissement, la souffrance et la réadaptation.

L'aspect communautaire ressort également de l'analyse des termes; il est au cœur de cinq numéros de Reflets. Du point de vue thématique, les questions du genre, de la famille, des femmes et de l'enfance se retrouvent dans cinq numéros alors que le terme "violence » est explicitement mentionné dans trois numéros et "paternités " dans deux numéros. Qui plus est, l'avant-dernier numéro de la revue (Vol. 21, No 1, printemps 2015) est consacré aux services en français en contexte minoritaire en matière de violences faites aux femmes, ce qui témoigne de sa mission et de ses principales préoccupations. D'ailleurs, il ressort clairement à la lecture des divers numéros que les groupes de femmes, les projets et recherches qu'elles mettent de l'avant et leurs revendications face aux inégalités structurelles de genre constituent un des apports les plus importants de la revue. 
"...nous notons une

panoplie d'angles

d'analyse, passant

des perspectives

structurelles,

féministes ou radicales

aux approches

psychosociales ou

systémiques. En ce sens, Reflets n'est pas porteur de couleurs idéologiques bien campées, mais plutôt d'un ensemble de perspectives articulées au fil de ses différents articles."
Des notions sémantiques pour le travail social comme " incapacité ", "vieillir ", " souffrance psychique et morale ", " réadaptation ", "spiritualité ", " développement économique ", " appauvrissement " et " exclusion » circonscrivent aussi l'intérêt thématique et au moins un numéro de la revue est consacré à chacune d'entre elles.

Soulignons par ailleurs que deux articles portent sur la résistance ou le refus comme formes d'intervention, soit celui de Welch (1995) et celui de Pinard et St-Amand (2013). À ce titre, il serait intéressant de se pencher sur les modèles d'analyses privilégiés par les auteures et auteurs des articles. Au premier abord, nous notons une panoplie d'angles d'analyse, passant des perspectives structurelles, féministes ou radicales aux approches psychosociales ou systémiques. En ce sens, Reflets n'est pas porteur de couleurs idéologiques bien campées, mais plutôt d'un ensemble de perspectives articulées au fil de ses différents articles.

Mentionnons finalement que la profession de travail social en Ontario fait l'objet de quelques numéros particuliers, un sur les approches féministes (Visibles et Partenaires : Pratiques et recherches féministes, Vol. 3, No 2, automne 1997), un sur les approches d'intervention en général (Approches d'intervention : définir et renouveler nos pratiques, Vol. 6, No 1, 2000), un autre sur le travail social en Ontario (Vol. 6, No 1, 2001) et un dernier sur les approches structurelles (Approches structurelles et intervention sociale (Vol. 19, No 1, printemps 2013),

lieux où on vise à présenter les dimensions complexes d'un travail social en contexte minoritaire. Ce numéro du printemps 2013 est le seul à être dédié à une personne en particulier, Roland Lecomte, cocréateur de l'École de service social de l'Université d'Ottawa et source d'inspiration dans le domaine du travail social structurel.

Au fil des articles publiés, nous constatons que l'intérêt pour le social reste toujours très présent. D'ailleurs, l'usage de l'adjectif " social " apparaît dix fois dans les trente-cinq titres des numéros publiés à ce jour, par exemple, Exclusion sociale (Vol. 11, No 1, 2005), Problèmes sociaux en Ontario français (Vol. 6, № 2, automne 2000) et 
L'intervention sociale en contextes minoritaires : penser la complexité et la multiplicité des processus de minorisation (Vol. 6, No 2, 2010). Dans ce dernier numéro en particulier, le titre rejoint trois thèmes chers à la revue : l'approche, l'intervention et le contexte minoritaire.

L'Annexe 3 présente tous les thèmes abordés depuis vingt ans, en ajoutant les noms des divers responsables de chacun des numéros. Celles et ceux qui connaissent l'univers minoritaire francophone constateront que plusieurs personnes, issues principalement du monde universitaire et des réseaux de recherche, ont accepté la responsabilité des différents numéros, et que certains noms, dont ceux de personnes ayant œuvré dans la revue depuis ses débuts, sont davantage présents. Cette liste des thèmes développés dénote certes une grande variété de perspectives et de sujets abordés en

«...Reflets ratisse large, car l'intervention se conjugue de nombreuses manières et sous plusieurs formes, ce qui fait en sorte que la revue est susceptible d'intéresser moult personnes et ressources dans les vastes domaines de l'intervention sociale." vingt ans. En ce sens, Reflets ratisse large, car l'intervention se conjugue de nombreuses manières et sous plusieurs formes, ce qui fait en sorte que la revue est susceptible d'intéresser moult personnes et ressources dans les vastes domaines de l'intervention sociale. Nous constatons aussi que pour la coordination des numéros la revue a souvent recours à des personnes n'appartenant pas à sa direction, ce qui dénote une grande solidarité des divers acteurs et actrices engagés dans sa mission et en confirme la vision initiale. Il y a lieu de souligner ici ce travail essentiel de la part de toutes ces personnes qui ont contribué de façon significative à l'impact de la revue et à son apport tout autant académique que professionnel et communautaire.

La rubrique Des pratiques à notre image (incluant l'ancienne Aux quatre coins de la province) présente pour sa part une très grande variété de sujets, de perspectives et d'angles d'analyse qui témoignent de la vitalité des expériences de terrain menées par un nombre important de personnes et de ressources œuvrant au sein de leurs communautés respectives. En voici quelques exemples :

- la médicalisation du travail social;

- la femme, sa sexualité et son pouvoir;

- les comités consultatifs jeunesse;

- le système des pauvres;

- la nouvelle économie sociale;

- les ailes francophones, un pas vers l'autonomie des femmes; 
"...les auteures on auteurs des Pratiques à notre image sont des personnes bien ancrées dans leurs communautés et elles représentent les services offerts dans leur coin de la province, ou ailleurs au pays. La rubrique permet de prendre contact avec l'épiderme des problèmes sociaux spécifiques aux populations minoritaires. "
- la marche mondiale des femmes;

- intervention et crise suicidaire;

- l'offre active de services;

- les ainés francophones et les intervenants multiethniques;

- les centres de santé communautaire;

- la formation à distance;

- la spiritualité et l'intervention sociale;

- la réadaptation communautaire;

- l'évolution de la profession de sage-femme;

- une campagne de consentement au Yukon.

Nous constatons que ces articles décrivent des problématiques ou projets plus pointus, tout aussi novateurs que colorés, tout en présentant l'intervention sous des angles remplis de dynamisme et de créativité. Par ailleurs, les auteures ou auteurs des Pratiques à notre image sont des personnes bien ancrées dans leurs communautés et elles représentent les services offerts dans leur coin de la province, ou ailleurs au pays. La rubrique permet de prendre contact avec l'épiderme des problèmes sociaux spécifiques aux populations minoritaires. De plus, il s'agit d'un excellent outil pédagogique et d'un forum important de partage d'information. Ces «lieux de passage " (Benjamin, 1939; [2003]) de la théorie à la pratique, sont axés tout autant sur des pratiques novatrices, des savoir-faire concrets que des préoccupations quotidiennes. En somme, ces fenêtres sur les milieux d'intervention complémentent les autres rubriques de la revue et en font une où l'expérience devient expertise partageable, mais aussi lieu commun de plusieurs conditions identitaires, qu'elles soient professionnelles, linguistiques ou de genre, entre autres.

\section{La complémentarité : le visuel et le textuel}

Lors des premières années d'existence de Reflets, les lectrices et lecteurs ont pu constater un désir de représentation visuelle sur sa page couverture. Diverses images, photos ou représentations graphiques ont illustré la page couverture des seize premiers 
numéros. Mais à compter de l'automne 2003 et jusqu'à maintenant, des contraintes logistiques et financières ont fait en sorte qu'on a cessé de présenter de façon imagée le contenu des articles ou des thèmes de chaque numéro, tout en restant fidèle à un cadrage graphique initial, jeu visuel autour du mot Reflets et en particulier sur la lettre " $\mathrm{R}$ » reprise et décalée (voir Figure 1). Tel qu'on l'observe dans cette couverture classique, marque de commerce et logo de la revue, son but et sa mission sont présentés à l'aide d'un carré-lentille à plusieurs dimensions superposées, ce qui suggère une représentation de l'intervention sociale qui se voit et s'amplifie, à l'image des communautés francophones minoritaires que Reflets représente. Nous constatons que la couverture reste sobre, n'ayant en plus de l'image que le titre du numéro et ses données de parution. La quatrième de page présente le contenu de la revue, soit ses diverses rubriques et les titres des articles qui la composent avec les noms de leurs auteures ou auteurs.

\section{Figure 1 - Couverture " classique" Reflets, Vol. 20, No 1, printemps 2014}

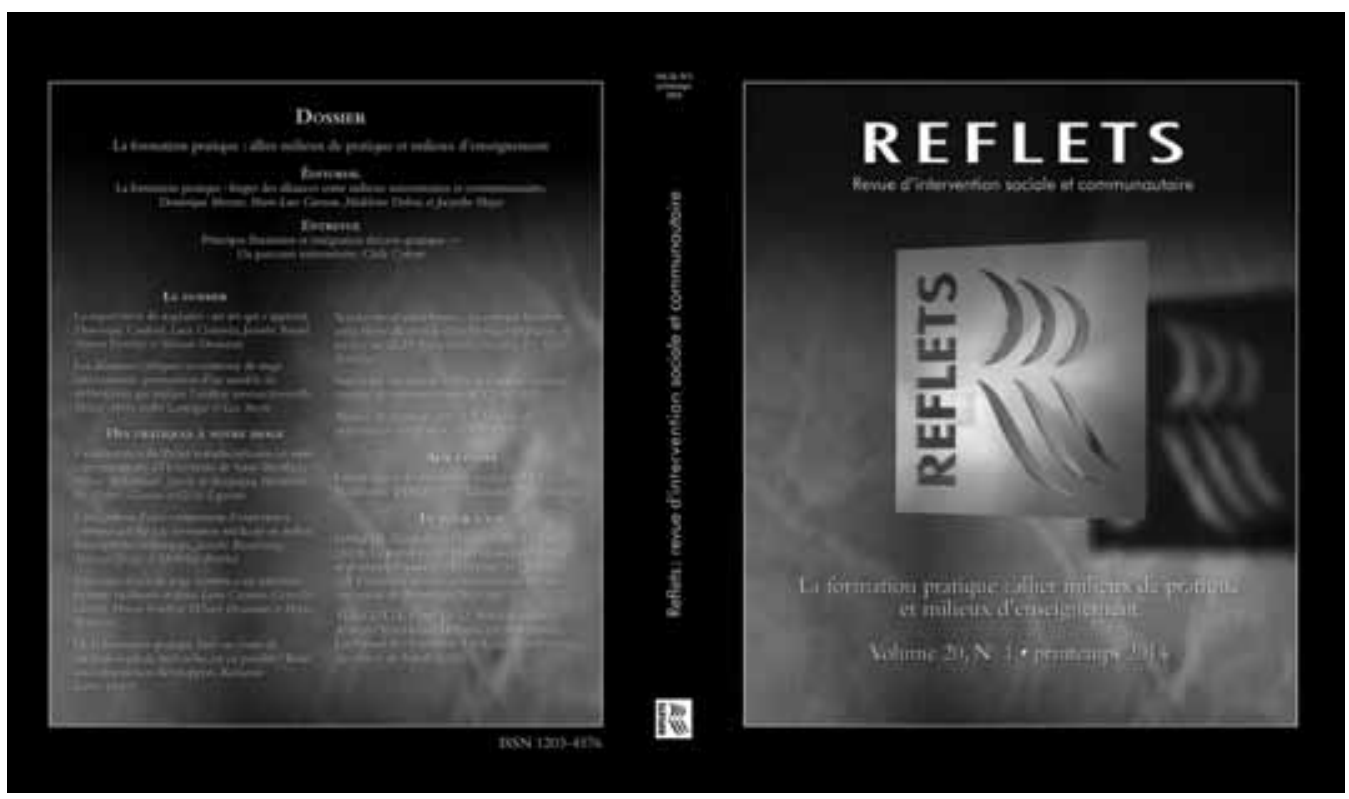


Par ailleurs, le numéro que vous feuilletez actuellement constitue une exception : sa page couverture présente la reproduction d'une des toiles gagnantes d'un concours initié par l'Association étudiante de l'École de service social (ADESS) de l'Université d'Ottawa. Reflets a d'ailleurs contribué financièrement au projet Créer pour lutter: l'art au service de la résistance. Ainsi, la mission sociale de la revue s'ouvre, ne fût-ce qu'exceptionnellement, sur une autre dimension :l'expression artistique et les représentations symboliques en contexte minoritaire.

Figure 2 Couverture Reflets, Vol. 21, № 2, automne 2015

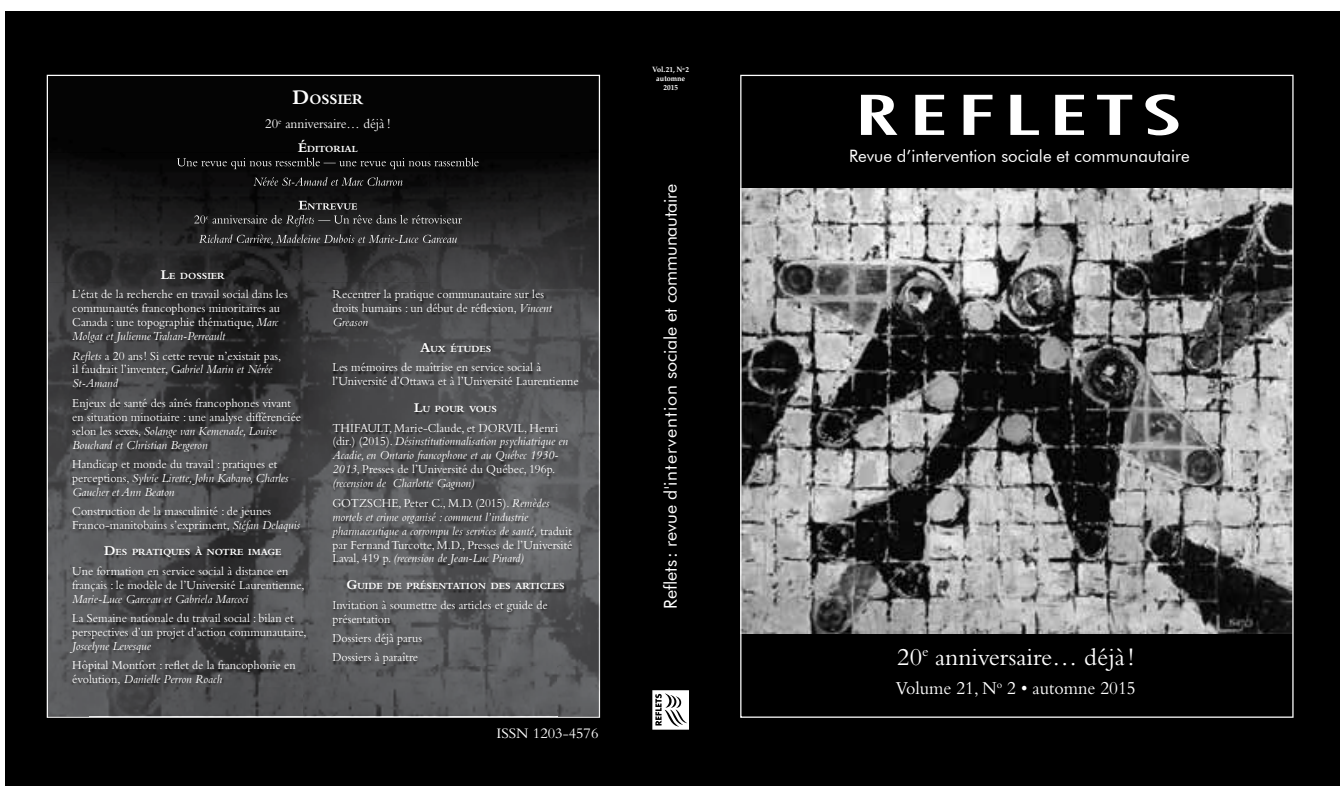

\section{Les entrevues : des mines d'or!}

Les entrevues constituent un apport important et original à la revue, chacune démontrant un choix judicieux de personnes qui 
"Les entrevues constituent un apport important et original à la revue, chacune démontrant un choix judicieux de personnes qui apportent un regard empreint de sagesse et d'expérience à chaque numéro. " apportent un regard empreint de sagesse et d'expérience à chaque numéro. Nous ne faisons que souligner certaines d'entre elles :

- Monique Bégin, ancienne ministre fédérale de la Santé et professeure émérite à l'Université d'Ottawa, présente ses réflexions sur l'orientation des programmes de santé (Vol. 5, No 2, automne 1999, p. 22-41);

- Wesley Romulus, directeur du centre médico-social communautaire de Toronto décrit les défis auxquels sont confrontés les centres communautaires (Vol. 1, No 2 , automne 1995, p. 18-36);

- Éthel Côté, directrice générale du Conseil de coopération de l'Ontario, partage ses réflexions sur le développement économique communautaire (Vol. 5, N 1, printemps 1999, p. 18-25);

- Louise Plouffe du Conseil consultatif national sur le $3^{\text {e }}$ âge aborde les liens entre autonomie et vieillissement (Vol. 2, No 2 , automne 1996, p. 16-35);

- Katy, femme autochtone, décrit l'impact intergénérationnel des pensionnats (Vol. 21, No 1, printemps 2015, p. 18-37);

- Antoine Dérose, citoyen canadien d'origine haïtienne et intervenant social, aborde les défis des nouveaux arrivants en Ontario (Vol. 4, No 1, printemps 1998, p. 20-31).

Grâce à l'Entrevue, le lectorat jouit donc d'une série de témoignages, d'opinions et de réflexions sur des sujets d'actualité, venant personnes qui ont marqué leur époque, leur champ de recherche ou leur contexte d'intervention.

\section{Les thèmes plus ou moins présents...}

Certes, l'étude des thèmes abordés ne dévoile pas l'ensemble du contenu des numéros, qui sont plus riches et qui présentent de nombreux volets sur des sujets complexes. Cela dit, qu'en est-il 
des thèmes moins abordés? Il nous semble que le fait de privilégier des numéros thématiques fait en sorte que la revue reste presque silencieuse sur certains sujets, dont :

- Les questions autochtones. Seulement deux articles (Sande, et Renault, 1998) et (Moeke-Pickering, et Partridge, 2014), de même qu'une entrevue (Vol. 21, No 1, printemps 2015) ont été publiés à ce jour. Nous concevons qu'il reste difficile de réaliser un numéro sur la francophonie autochtone ou encore, sur celle des Métis, et ce, même dans les revues à plus fort tirage publiées en français, en raison du fait qu'outre leur langue d'origine, les personnes des Premières Nations ou les Métis emploient plus souvent l'anglais dans leurs transactions avec les Blancs;

- La pauvreté. Ce sujet a été abordé en 1997 sous le titre Enfance et famille en contexte d'appauvrissement (Vol. 3, $\mathrm{N}^{\circ}$ 1, printemps 1997) et ayant fait l'objet de la première entrevue en 1995 avec Lynne Toupin au sujet de l'économie sociale et solidaire et d'un numéro sous le thème Pratiques et développement économique communautaire (Vol.1, No 1, printemps 1995). Nous notons par ailleurs que cette problématique transcende certains autres thèmes, en particulier ceux des femmes et de la santé mentale;

- La question des droits sociaux, des droits des minorités, des personnes et groupes délaissés, et par ricochet, des pratiques de solidarité;

- l'écologie sociale et ses liens avec l'intervention sociale;

- l'interdisciplinarité en travail social;

"Ces thèmes pourraient inspirer la création de numéros spéciaux de Reflets et l'ouvrir ainsi à d'autres univers culturels et politiques de l'intervention en contexte minoritaire."
- Les actrices et acteurs qui ont fait évoluer le travail social et l'intervention sociale en contexte francophone minoritaire;

- Les formes et stratégies d'intervention privilégiées dans les milieux de pratiques institutionnalisés;

- Les recherches et interventions comparées avec d'autres espaces géographiques et avec d'autres populations minoritaires vivant dans des situations politico-géographiques semblables.

Ces thèmes pourraient inspirer la création de numéros spéciaux de Reflets et l'ouvrir ainsi à d'autres univers culturels et politiques de l'intervention en contexte minoritaire. 


\section{4 : Reflets se joint à la plateforme Érudit}

"Depuis 2004, Reflets fait partie du consortium Érudit. Cette plateforme de diffusion numérique permet une très large diffusion de l'ensemble de son contenu..."
Depuis 2004, Reflets fait partie du consortium Érudit. Cette plateforme de diffusion numérique permet une très large diffusion de l'ensemble de son contenu, en plus de prendre en charge la gestion des abonnements. Cette décision de faire partie du consortium, alors que celui-ci n'en était qu'à ses débuts, a augmenté considérablement la visibilité de la revue et l'accessibilité aux textes publiés. Depuis, Reflets présente en français et en anglais le résumé de ses articles arbitrés, et ce, aux fins des moteurs de recherche de la plateforme. Érudit fournissant régulièrement des statistiques d'utilisation, en voici quelques-unes qui donnent un aperçu de la visibilité de la revue :

- entre 2008 et 2014, les publications de Reflets ont reçu plus de 280000 visiteurs différents;

- les visiteurs de Reflets proviennent principalement, en ordre de fréquence, du Canada, de la France, des États-Unis, de l'Allemagne, de la Chine, du Danemark, de la Suisse et de la Belgique;

- le nombre de visiteurs est passé de 50531 en 2008 pour se stabiliser autour de 27546 en 2014;

- en 2014, le nombre de pages vues (hits) variait entre 7000 à 12000 par mois, les mois de mars et d'avril étant les plus achalandés.

Sur le plan des abonnements, plusieurs formules existent, de l'abonnement individuel au "panier", soit un ensemble de revues. Nous constatons qu'en 2015, ce type d'abonnement se retrouve principalement au Québec, puis en Ontario et dans d'autres provinces et d'autres pays. Reflets est utilisé principalement au niveau collégial et universitaire, dans les formations de premier et de second cycle.

La recherche effectuée par Molgat et Trahan-Perreault (2015) publiée dans le présent numéro témoigne du rôle central qu'occupe Reflets au sein des publications scientifiques, surtout 
en ce qui concerne l'Ontario français, mais aussi en contexte minoritaire canadien.

\section{Un sondage révélateur...}

"Les commentaires sont unanimes : la revue reflète la réalité des actrices et acteurs d'une francophonie minoritaire, que ce soit dans les domaines de la recherche sociale, de la formation académique ou professionnelle tout autant que de l'intervention sous toutes ses formes, qu'elle soit classique, féministe, structurelle, spirituelle ou alternative. »
Cette analyse ne serait pas complète sans la voix des lectrices et des lecteurs de Reflets : revue d'intervention sociale et communautaire, soit les chercheuses et chercheurs, intervenantes et intervenants des domaines social et communautaire en contexte francophone minoritaire. À l'heure du bilan, nous avons voulu ouvrir une forme de dialogue, fort limité il va sans dire, avec celles et ceux qui ont contribué à la création et à l'évolution de Reflets, pour ainsi en évaluer la portée, les impacts et poser la question de son orientation future. En somme, nous désirions simplement offrir un lieu de parole aux quelque vingt-cinq personnes qui ont contribué à façonner la revue, soit dans sa conception, soit dans ses orientations. Nous leur avons demandé de répondre à un bref questionnaire en ligne. Bien qu'il ne s'agisse pas d'un exercice scientifique, les commentaires qu'on y retrouve dénotent l'importance de la revue, particulièrement quant à ses impacts ou aux différents acteurs et actrices qui gravitent autour de sa mission.

\section{Reflets, une nécessité}

Tous les répondantes ou répondants témoignent de la portée de Reflets, en 1995 certes, mais encore davantage à mesure que le temps en façonne son identité propre. Les commentaires sont unanimes : la revue reflète la réalité des actrices et acteurs d'une francophonie minoritaire, que ce soit dans les domaines de la recherche sociale, de la formation académique ou professionnelle tout autant que de l'intervention sous toutes ses formes, qu'elle soit classique, féministe, structurelle, spirituelle ou alternative.

"À l'origine, la revue Reflets voulait combler un manque de pertinence des revues québécoises en raison de leur choix éditorial privilégiant l'examen de réalités du Québec.» 
"Reflets est au cœur du rayonnement de la recherche en contexte francophone minoritaire.»

"Pour nos enseignements, il s'agit d'une des seules revues où on peut trouver des textes sur le travail social en contexte minoritaire. »

"Il n'y a pas un cours où je n'utilise pas des articles de Reflets. C'est la valeur ajoutée de Reflets : un outil pédagogique calibré aux réalités francophones minoritaires. "

"La revue se veut une intervention en soi en créant un savoir propre aux minoritaires, un savoir où les personnes se connaissent et se reconnaissent, d'où le titre de la revue."

En somme, toutes les réponses font état des impacts significatifs de la revue depuis vingt ans. La pérennité de Reflets est le signe manifeste qu'elle répondait et répond toujours à un besoin.

\section{Reflets et contexte minoritaire}

La revue a su rassembler les divers acteurs et actrices dans le domaine francophone minoritaire, être la voix de grands projets qui se sont déroulés au cours des derniers vingt ans, offrir des écrits de belle qualité aux intervenantes et intervenants, aux professionnelles et professionnels, aux étudiantes et étudiants, aux universitaires et enfin, constituer un élément déclencheur de plein d'initiatives dynamiques dans les communautés francophones minoritaires. Et une répondante de conclure :

«Bravo! Si cette revue n'existait pas, il faudrait

" «Bravo! Si cette

revue n'existait pas, il faudrait la créer! " "

Toutes et tous confirment le rôle d'une revue d'intervention sociale pour les personnes engagées en recherche ou en intervention, ces témoins, acteurs et actrices de leur condition minoritaire, qui sentent le besoin de répondre aux nécessités sociales de leur communauté. 
"Toutes et tous

soulignent que la revue a contribué à bâtir non seulement une expertise spécifique, mais aussi une identité professionnelle francophone; de la sorte, elle a contribué à rassembler les soucis identitaires des communautés de la francophonie minoritaire. "
«Reflets a largement contribué à la diffusion de la recherche en Ontario français et dans d'autres contextes minoritaires, milieux géographiquement dispersés, parfois isolés et ayant peu d'accès à des possibilités de publication. "

Une répondante souligne ainsi la précarité de la représentativité sociale francophone minoritaire de l'époque dans l'ensemble de la société canadienne :

«Avant 1995, il n'y avait aucune revue scientifique traitant des problèmes et des enjeux spécifiques à la pratique du travail social en milieu minoritaire francophone en Ontario.»

\section{Reflets et identité professionnelle}

Toutes et tous soulignent que la revue a contribué à bâtir non seulement une expertise spécifique, mais aussi une identité professionnelle francophone; de la sorte, elle a contribué à rassembler les soucis identitaires des communautés de la francophonie minoritaire.

"En 1990, lors de mon arrivée en Ontario, nous n'avions aucun texte scientifique propre à l'Ontario français ou au contexte francophone minoritaire. Nos références venaient du Québec et de la France. Maintenant, tant au niveau des recherches scientifiques que des projets communautaires, nous avons des centaines de possibilités à notre portée. De plus, le consortium Érudit a contribué à une large diffusion des articles de la revue.»

Une répondante se penche davantage sur la portée de Reflets sur le plan de l'intervention :

"Reflets constitue aussi un outil d'intervention dans la mesure où il éveille les milieux minoritaires à des réalités qui ne sont pas nécessairement 
" " C'est une revue qui est unique en Amérique du Nord par le fait même qu'elle représente un espace de débats et de discussions académiques et de réflexions sur les pratiques d'interventions sociales dans le contexte francophone minoritaire, une revue comme il n'y a pas au Canada." " traitées, discutées dans ces mêmes milieux. Cette revue agit en avant-garde, cherchant certes à mobiliser, mais également à interroger les communautés minoritaires autour d'enjeux et de questions qui ne vont pas nécessairement de soi dans ces communautés. »

La revue fait ainsi figure d'acteur politique; c'est un outil d'empowerment social :

«La revue encourage l'échange, le partage, pour les francophones qui vivent des situations de pauvreté et de marginalité qu'on a autrement tendance à passer sous silence. On ne peut pas s'adresser aux problèmes du présent de cette communauté sans reconnaitre le contexte historique du développement du service social en Ontario français et sur lequel s'appuient les nouvelles pratiques et les innovations dans les domaines du service social et de la santé.»

C'est pourquoi, opine une autre répondante, la revue Reflets est unique en son genre :

"C'est une revue qui est unique en Amérique du Nord par le fait même qu'elle représente un espace de débats et de discussions académiques et de réflexions sur les pratiques d'interventions sociales dans le contexte francophone minoritaire, une revue comme il n'y a pas au Canada.»

Une autre souligne le rôle de diffuseur continuel d'articles, rôle que Reflets a assumé à travers les années, s'inscrivant dans une démarche de démocratisation de la communication sociale jusqu'alors monopolisée par certains acteurs privilégiés, loin des préoccupations locales et informelles.

"Je pense à des numéros sur la violence contre les femmes : ils ont permis de diffuser ce qui se fait dans le domaine, ici, en Ontario français. Les numéros sur la santé vont dans le même sens : 
ils abordent les questions du point de vue des populations en milieux minoritaires et comblent ainsi un vide entre les réseaux de communication formels et les lieux de production de savoir local, informel.»

\section{Un rôle élargi pour la revue}

Plusieurs répondantes ou répondants émettent des suggestions en faveur d'un rôle encore plus dynamique, plus interactif, plus vaste pour la revue. Par exemple, quel rôle pourrait jouer Reflets auprès des gouvernements au niveau municipal, provincial ou fédéral dans la planification, dans la prise de décision ou dans la gouvernance sociale? Dans quelle mesure la revue a-t-elle influencé les politiques sociales et autres lieux de changement institutionnel? En créant un bassin de chercheuses et de chercheurs qui se penchent sur les réalités minoritaires, la revue Reflets a certes influencé, ne seraitce qu'indirectement, les gouvernants en mettant à disposition un ensemble de données et de réflexions qui alimentent le débat public, notamment, en matière de violence faite aux femmes, de santé des francophones vivant en Ontario et de services en français. Toutefois, cette proximité des niveaux institutionnels peut aussi servir de mise en garde à la revue, car un danger de conformité ou encore d'inertie pourrait en faire oublier la mission :

"Reflets s'est trop aligné aux pratiques institutionnelles et n'a pas su garder une distance critique face aux institutions de formation, de recherche et d'intervention. C'est en ce sens que certains thèmes essentiels ont pratiquement été évités, par exemple la pauvreté et les très grandes et grandissantes inégalités sociales, entre régions et groupes de populations, particulièrement les plus vulnérables. Est-ce que ceci révèle un engagement tiède de la part de la revue, reflétant un positionnement équivoque face à un état de moins en moins providentialiste et une profession jouant le jeu d'un conformisme progressiste? » 
"Quant au renouvellement de sa mission, une nouvelle ouverture prend forme, soit celle vers d'autres horizons francophones - tant au Canada que sur le continent nord-américain et ailleurs dans le Monde."
Quant au renouvellement de sa mission, une nouvelle ouverture prend forme, soit celle vers d'autres horizons francophones tant au Canada que sur le continent nord-américain et ailleurs dans le Monde.

«Il serait peut-être pertinent d'élargir le comité de direction ou de créer un comité scientifique international. »

Par cette ouverture à l'international, on vise aussi le terrain d'expérience de pratiques :

"Je suggérerais d'ouvrir la revue à des auteurs et problématiques de travail social d'autres régions minoritaires du monde.»

D'autres commentaires visent diverses formes d'élargissement des perspectives, dans le sens où «la place des articles hors thèmes pourrait aussi être revue » et présenter ainsi un plus grand éventail d'idées que celles qui sont généralement planifiées un an ou deux à l'avance. Par ailleurs, quelqu'un suggère que le site You Tube soit utilisé pour diffuser l'intégral des entretiens présentés dans l'entrevue. Enfin, l'élément inclusif des nouvelles générations préoccupe également plusieurs répondantes et répondants :

"L'ouverture à la nouvelle génération, qu'on offre la chance de passer la relève de la revue à des jeunes futurs travailleurs et futures travailleuses sociales. »

De ce point de vue Reflets pourrait aussi être un instrument intergénérationnel accessible aux jeunes. La revue devrait être ouverte aux étudiantes et étudiants, souligne le sondage, " pour qu'ils puissent publier leurs recherches ".

Une autre personne renchérit :

«Il faudrait que Reflets permette à des doctorantes ou doctorants de diriger des numéros, donnant ainsi une vraie place aux étudiantes et étudiants des deuxième et troisième cycles ». 
"Il faut continuer cet excellent travail d'agir comme rassembleur et diffuseur des recherches et des pratiques qui se font dans les milieux francophones minoritaires. »
Nous notons par ailleurs que quelques étudiantes ou étudiants inscrits à une maitrise ou à un doctorat en service social ont déjà codirigé ou codirigent des numéros de la revue.

\section{Dans un axe de continuité et de diversification}

Dans l'ensemble, les personnes répondant au questionnaire formulent des vœux de continuité et de diversification de ses formes de diffusion :

"Il faut remercier les artisans de la revue et reconnaitre le travail très important qu'ils ont fait pour la développer et remercier également les bailleurs de fonds. Il faut continuer cet excellent travail d'agir comme rassembleur et diffuseur des recherches et des pratiques qui se font dans les milieux francophones minoritaires.»

\section{Que conclure? Ou plutôt : faut-il conclure?}

En somme, Reflets reste une histoire à suivre. En ce sens, nous ne proposons pas de conclusion comme telle, mais une ouverture au monde. Il semble évident à la suite de cette brève analyse que la revue a joué et joue encore un rôle important, capital même, dans l'évolution de l'intervention sociale en contexte minoritaire, en Ontario certes, mais aussi ailleurs dans des milieux de formation, de recherche et d'intervention. En voici certains volets :

\section{Reflets s'est taillé une place}

Il y a vingt ans, Reflets s'est créé une niche et a graduellement pris une place bien campée dans le vaste et riche univers de l'intervention sociale en contexte minoritaire. La revue fait maintenant partie des outils d'information, d'échange, de formation, de recherche et de mobilisation, ces éléments qui définissent le travail social en ce $21^{\mathrm{e}}$ siècle. Sa crédibilité n'est plus à établir, tant dans les cercles universitaires que dans les lieux d'intervention, qu'ils soient institutionnels, communautaires ou alternatifs. Qui plus 
"Il $y$ a vingt ans, Reflets s'est créé une niche et a graduellement pris une place bien campée dans le vaste et riche univers de l'intervention sociale en contexte minoritaire. La revue fait maintenant partie des outils d'information, d'échange, de formation, de recherche et de mobilisation..." est, sa présence au sein du Consortium Érudit en particulier lui assure un rayonnement international.

Étant donné sa base relativement solide, tant en matière de ressources que de gestion, la revue pourrait faire plus, élargir ses perspectives et être présente à diverses autres instances pour faire évoluer le travail social - et aussi les autres professions d'aide dans le sens des droits, de l'égalité, du renouvellement des pratiques. Pour ce faire, une place de choix pourrait être accordée à une réflexion sur ce qu'est le travail social, basée sur des valeurs qui représentent la profession dans ses formes les plus conscientisantes. En effet, si les horizons de l'intervention ne doivent pas se limiter aux approches institutionnelles et aux visions professionnelles qui ont souvent tendance à adopter des perspectives limitées de ce que peuvent faire les professions d'aide, Reflets doit continuer d'élargir le regard et les possibilités en s'inspirant de personnes et de réseaux qui ont ennobli le social et inspiré ses actrices et acteurs.

À cette heure de bilan, force est de constater que plusieurs des artisanes et des artisans de la première heure se sont retirés ou le feront prochainement. La relève nous semble capitale à cette étape, qu'elle soit recrutée chez les étudiantes ou les étudiants, au sein des personnes représentant les communautés de pratique ou dans les divers lieux de formation. Une ouverture à l'échelon national et international, la revue ayant été présente à des forums nationaux (ACFAS et ACFTS) et à un forum international (AIFRIS, Portugal, été 2015), nous semble ici une occasion rêvée de concrétiser les lieux et formes de réflexion que Reflets a déjà bien entamés.

\section{Créer des liens...}

La revue a créé des liens synergiques entre divers milieux de pratique et de nombreux secteurs de recherche et de formation. Cela dit, il nous semble que davantage de liens sont à établir entre les diverses problématiques présentées. Depuis les débuts, chaque numéro semble avoir constitué une entité en soi, plus ou moins distincte des autres, sans que se tissent des liens structurels entre les numéros et entre les problématiques. Une fois le numéro 
terminé, on passe au suivant tout en n'ayant pas nécessairement fait le tour de questions complexes. Par exemple, comment étudier la santé mentale sans y adjoindre les problématiques de pauvreté? Comment parler des jeunes ou des personnes vieillissantes sans aborder les politiques sociales et les politiques d'habitation? Et comment présenter les réalités autochtones sans parler de genre, d'exclusion sociale, des pratiques innovantes ou de formation? En ce sens, si la revue continue de planifier en fonction de thématiques et de personnes responsables de chacune, la question se pose : comment faire davantage de liens entre les problématiques, tenant compte évidemment de la dimension minoritaire?

\section{Reflets, au féminin!}

À la lecture de cette richesse d'information contenue dans la revue, nous soulignons l'immense contribution des femmes tout au long de ses vingt ans. Tant sous l'angle de l'écriture que de la participation et de l'engagement dans de nombreux dossiers, il se dégage de notre étude de vingt ans de publications une présence féminine marquée, soutenue, empreinte de solidarités, faite d'un engagement sans relâche, d'un investissement dans le présent et dans l'avenir de la revue, en lien évidemment avec la survie des populations francophones minoritaires. Ces femmes qui s'engagent et qui publient témoignent de convictions profondes, tant idéologiques que pratiques, amenant du même coup une sensibilité, une recherche d'alternatives et quelques lueurs d'espoir face aux problèmes sociaux actuels. Grâce à vous, femmes du $21^{\mathrm{e}}$ siècle, la bataille des épingles à chapeaux prend un autre tournant d'engagement!

\section{Reflets se souvient}

L'histoire se souviendra de nombreuses personnes et ressources qui ont contribué à l'essor de la revue, que ce soit les artisanes et artisans de la première heure, les ministères et organismes qui ont généreusement appuyé la revue - dont l'Université Laurentienne, l'Université d'Ottawa et le Consortium national de formation en santé (CNFS) - tout en n'oubliant pas les 
personnes qui ont effectué dans l'ombre le travail quotidien de gestion des nombreux dossiers, soit à l'Université d'Ottawa, soit à l'Université Laurentienne ou encore à Media Concepts Médiatiques Inc. de Sudbury, ressource responsable de la mise en page et de l'édition depuis la première heure. Soulignons le travail de révision linguistique réalisé par André Dubois depuis les premiers numéros de Reflets et celui de Jeannine Turpin qui veille fidèlement au rouage de l'entreprise. Enfin, nous désirons rendre un hommage spécial à une artisane de la première heure, Michèle Kérisit, décédée le 23 juin 2011. Féministe dévouée, elle était professeure à l'École de service social de l'Université d'Ottawa.

À toutes ces personnes artisanes d'un avenir plus inclusif et égalitaire : Reflets se souvient et lève son chapeau!

\section{Remerciements}

Nous désirons remercier Marie-Luce Garceau et Marc Charron pour leur grande collaboration dans la préparation de cet article.

\section{Notes}

1 Sur le plan individuel, sur le rôle de la querelle, voir les contributions d'Y. Bernthard et George Bach. Sur le plan des collectivités, voir les querelles des élites pour construire un "état vertébré " au sens de España invertebrada [Espagne vertébrée] de José Ortega y Gasset.

2 Voir à titre d'exemple le numéro de la Revue de l'Université de Moncton, Vol. 33, № 1-2, 2002, consacré au thème de "L'Économie sociale et solidaire ». Écrite en "langue française " par des auteurs "provenant de la communauté universitaire régionale, nationale et internationale ", "pluridisciplinaire et humaniste ", elle est publiée deux fois par année et « ouvre ses pages à des collaborations externes ». (Endos de couverture)

3 Pour Ricœur (2000), l'historien s'avère être un thérapeute social; il voit son rôle comme essentiel, car il libère la mémoire collective du fardeau honteux de son passé. 


\section{Bibliographie}

ARENDT, Hannah (1955; [2002a]). Les origines du totalitarisme [suivi de] Eichmann à Jérusalem, édition établie sous la direction de Pierre Bouretz, Paris, Gallimard, collection Quarto, 1620 p.

ARENDT, Hannah (1955; [2002b]). Les origines du totalitarisme, tome 2, L'impérialisme, Paris, Seuil, $378 \mathrm{p}$.

ARENDT, Hannah (1958). Condition de l'homme moderne, Paris, Calmann-Lévy, collection Pocket Agora., $416 \mathrm{p}$.

BACH, George Robert, et Peter WYDEN (1983). Intimate enemy: How to fight fair in love and marriage, Detroit, Michigan, Motor City Books, 384 p.

BELLAH, Robert N., et collab. (c1985). Habits of the heart: individualism and commitment in American life, Berkeley, University of California Press, $410 \mathrm{p}$.

BENEDICT, Anderson (1983). Imagined communities, London, New York, Verso, 224 p.

BENJAMIN, Walter (1939; [2003]). «Paris, capitale du XIX siècle », Paris, Éditions Allia, 50 p.

BERNSTEIN, Richard (2005). " Hannah Arendt on the stateless ", dans Paralax, special issue on Seeking Asylum, New York, Routledge,Vol. II, No 1, p. 46-60.

BOCK, Michel (2001). Comment un peuple oublie son nom. La Crise identitaire franco-ontarienne et la presse française de Sudbury, 1960-1975, Sudbury, Prise de parole et Institut franco-ontarien, $119 \mathrm{p}$.

BOCK, Michel (2008). « Se souvenir et oublier : la mémoire du Canada français, hier et aujourd'hui ", dans Joseph Yvon Thériault, Anne Gilbert et Linda Cardinal (dirs.), L'espace francophone en milieu minoritaire au Canada. Nouveaux enjeux, nouvelles mobilisations, Montréal, Les Éditions Fides, p.161-203.

BOCK, Michel, et Gaétan GERVAIS (2004). L'Ontario français des Pays-d'en-Haut à nos jours, Ottawa, Centre de ressources pédagogiques de l'Ontario, $271 \mathrm{p}$.

BRETON, Raymond (1964). «Institutional Completeness of Ethnic Communities and the Personal Relations of Immigrants ", American Journal of Sociology, Vol. 70, №. 2, Sep., 1964, p. 193-205.

CARDINAL, Linda, et Eloisa GONZÀLEZ HIDALGO (2012). L'autonomie des minorités francophones hors Québec au regard du débat sur les minorités nationales et les minorités ethniques ", Minorités linguistiques et société / Linguistic Minorities and Society, No 1, 2012, p. 51-65.

CARRIÈRE, Richard (1995). "Présentation - Reflets : revue ontaroise d'intervention sociale et communautaire ", Reflets : revue ontaroise d'intervention sociale et communautaire, Vol. 1, $\mathrm{N}^{\circ} 1$, printemps, p. 1-8.

CASTEL, Robert (1994). «La dynamique des processus de marginalisation : de la vulnérabilité à la désaffiliation ", Cahiers de recherche sociologique, $\mathrm{N}^{\circ} 32$, p. 11-27.

CHARTIER, Roger (1982). "Intellectual History or Socio-cultural History? The French Trajectories ", dans Dominick LaCapra and Steven L. Kaplan, Modern European Intellectual History Reappraisals and new perspectives, Cornell University Press, p. 13-46.

CENTRE DE RECHERCHE EN CIVILISATION CANADIENNE-FRANÇAISE (CRCCF) (2004). «La présence française en Ontario : 1610, passeport pour 2010. Revendications et luttes scolaires ", réf. du 10 mai 2015, http://www.crccf.uottawa.ca/passeport/IV/IVD.html.

CHOQUETTE, Robert (1977). Langue et Religion. Histoire des conflits anglo-français en Ontario, Ottawa, Les Éditions de l'Université d'Ottawa.

DE CERTEAU, Michel (1980). L'invention du quotidien, Paris, U.G.E, 349 p. 
DERRIDA, Jacques (1967). L'Écriture et la différence, Paris, Seuil.

DIALLO, Lamine, et Marge REITSMA-STREET (1995). "Stratégies de survie et d'identité : les dynamiques culturelles dans un projet d'intervention en prévention communautaire ", Reflets : revue ontaroise d'intervention sociale et communautaire, Vol. 1, No 1, printemps, p. 43-69.

DUBOIS, Madeleine, et Nérée ST-AMAND (1995). "Des pratiques à notre image : défis et ressources ", Reflets : revue ontaroise d'intervention sociale et communautaire, Vol.1, No 1, p. xvii-xx.

FOUCAULT, Michel (1966). Les Mots et les Choses. Une archéologie des sciences humaines, Paris, Gallimard, coll. «Bibliothèque des sciences humaines »., 400 p.

FOUCAULT, Michel (1994). Dits et écrits, Paris, Gallimard, 864 p.

FREUD, Sigmund (1970). La Technique psychanalytique, Paris, PUF, 168 p.

GRISÉ, Yolande (1982). "Ontarois : une prise de parole ", Revue du Nouvel-Ontario, № 4, 1982, p. 81-82.

HARTOG, François (2003). Régimes d'historicité. Présentisme et expériences du temps, Seuil, La librairie du XX ${ }^{\text {e }}$ siècle, $271 \mathrm{p}$.

LEDUC-BROWNE, Paul (1995). "Déjà vu: Thatcherism in Ontario ", dans Diana Ralph, André Régimbald et Nérée St-Amand (dirs.), Open for Business, Closed to People, Halifax, Firnwood Publishing, p. 37-39.

LIANOS, Michalis (2006). "Le contrôle social après Foucault ", Surveillance E Society Vol 1, № 3, p. 431-448, réf du 16 mars 2015,

http://www.surveillance-and-society.org/articles1(3)/ApresFoucault.pdf

MA MUNG, Emmanuel (1999). "La dispersion comme ressource spatiale », Cultures et conflits, № 42, p. 43.

MOEKE-PICKERING, Taima, et Cheryle PARTRIDGE (2014). " Service social autochtone - Incorporer la vision autochtone du monde dans les stages pratiques en service social", Reflets : revue d'intervention sociale et communautaire,Vol. 20, № 1, p. 150-169.

MOLGAT, Marc, et Denise LEMIRE (1995). «Des jeunes à l'écart du social? Histoires des jeunes francophones sans abri à Ottawa-Carleton ", Reflets : revue ontaroise d'intervention sociale et communautaire, Vol. 1, No 1, p. 141-163.

MOLGAT, Marc, et Julienne TRAHAN-PERREAULT (2015). «L'état de la recherche en travail social dans les communautés francophones minoritaires au Canada : une topographie ", Reflets : revue d'intervention sociale et communautaire, Vol. 21, № 2, [présent numéro].

MAR GALIT, Avishai (2002). The Ethics of Memory, Cambridge, Harvard University Press, 240 p.

NORA, Pierre (dir.) (1997). Les lieux de mémoire, Les lieux de mémoire, tome 3, Paris, Gallimard, 986 p.

ORTEGAY GASSET,José (1921; [1992]). España invertebrada, Mexico, [édition de, Liga de Educación Política, $143 \mathrm{p}$.

PARÉ, François (1994). «L'Institution littéraire franco-ontarienne et son rapport à la construction identitaire des Franco-Ontariens ", dans Jocelyne Létourneau et Roger Bernard (dirs.), La question identitaire du Canada francophone, Récits, parcours, enjeux, hors lieux, Sainte-Foy, Les presses de l'Université Laval, p. 45-62.

PINARD, Jean-Luc, et Nérée ST-AMAND (2013). "De la conformité au refus : analyse d'un parcours ", Reflets : revue d'intervention sociale et communautaire, Vol.19, № 1, p. 85-118.

RICCEUR, Paul (2000). La mémoire, l'histoire, l'oubli, Paris, Éditions du Seuil, 672 p. 
RICCEUR, Paul (2002). "Mémoire : approche historienne, approche philosophique ", Le débat, № 122, nov-déc, p. 41-61.

RICCEUR, Paul (2004). Parcours de la reconnaissance, Paris, Gallimard, Ed. Stock., 386 p.

RIGNEY, Ann (1992). "The point of stories: On narrative communication and its cognitive functions ", Poetics Today, Vol. 13, № 2, Duke University Press, p. 263-283.

ST-AMAND, Nérée (2011). Repenser le service social?, Ottawa, Merriam Print, 324 p.

TARRIUS, Alain (2002). La mondialisation par le bas. Les nouveaux nomades de l'économie souterraine, Paris, Balland, $168 \mathrm{p}$.

THÉRIAULT, Joseph Yvon (2014). Complétude institutionnelle : du concept à l'action, réf. du 29 juin 2015, Cahiers du MIMMOC, Cahier 12, Cahier électronique, http://mimmoc.revues.org/.

VAN DE SANDE,Adje, et Gilles RENAULT (1998). «L'intégration des concepts autochtones dans le curriculum du travail social ", Reflets : revue ontaroise d'intervention sociale et communautaire, Vol. 4, No 1, p. 164-173.

WELCH, David (1995). «Les Franco-Ontariens : la résistance comme mode de vie ", Reflets : revue ontaroise d'intervention sociale et communautaire, $\mathrm{Vol}$ 1, No 1, p. 20-42.

WHITE, Hayden (1987). The content of the form: Narrative discourse and historical representation, Baltimore et London, Johns Hopkins University Press, 264 p. 


\section{Opposition au Règlement 17 en Ontario}

En 1912, le gouvernement de l'Ontario adopte le Règlement 17 qui interdit qu'on enseigne et même qu'on parle français dans les écoles ontariennes. Les hommes défendent les droits des francophones par le discours politique. Par exemple, le Père Charlebois crée le journal Le Droit et Louis-Philippe Landry démissionne avec fracas de son poste de président du Sénat canadien pour se consacrer à la présidence de l'Association canadienne-fr... ançaise d'éducation de l'Ontario (l'ACFO d'aujourd'hui). Il dénoncera, en 1917, l'œuvre subversive de l'évêque irlandais Fallon et blâmera aussi l'archevêque anglophone d'Ottawa. Son fauteuil de sénateur est aujourd'hui aux bureaux de l'ACFO où il est l'apanage du président. Samuel Genest, président de la Commission des écoles séparées d'Ottawa, pendant toute la crise du Règlement 17 (19121927), sera traîné devant les tribunaux et accusé d'avoir rémunéré des enseignants. Il sera aussi président de L'ACFÉO. La crise scolaire atteint son paroxisme. Monseigneur Élie Latulipe de Haileybury et d'autres évêques et dirigeants francophones appuient l'ACFÉO. Mais les femmes décident de défendre leurs droits en passant à l'action! Les enseignantes Diane et Béatrice Desloges sont expulsées de leur école Guigues à Ottawa pour avoir continué d'enseigner en français. Forcées de quitter l'école Guigues, les enseignantes Diane et Béatrice Desloges ouvrent des classes privées. La Commission des écoles séparées d'Ottawa veut reprendre l'école Guigues, mais les policiers leur barrent la route. En janvier 1916, les deux enseignantes et dix-neuf mères de famille reprennent possession de leur école. Les policiers encerclent l'édifice, mais les femmes montent la garde avec leurs fameuses épingles à chapeau. Les femmes occupent l'école pendant des semaines et continuent d'enseigner en français aux enfants. Puis, trente policiers défoncent la porte pour essayer de reprendre l'école avec leurs matraques. Ils sortent en courant et abandonnent après avoir reçu leur part de coups de rouleaux à pâte, de poêles de fonte et d'épingles! Ce jour-là, les Gardiennes de l'École Guigues ont fait un geste qui a inspiré tout l'Ontario à défendre l'éducation de langue française. D'autres femmes continueront le combat, telles Florence Quesnel à Green 
Valley, Anne-Marie Lemelin à Welland et Jeanne Lajoie à Pembroke. La crise scolaire se résorbe en 1921. Le Règlement 17 devient inopérant en 1927 et sera officiellement abrogé par le gouvernement qu'en 1944. Cette loi infầme aura toutefois permis aux Franco-Ontariens de se regrouper et de s'affirmer comme collectivité. (http://echo. franco.ca/guerredesepingles/index.cfm?Id $=32864 \&$ Sequence No $=\&$ Voir $=$ journal)

\section{Revendications et luttes scolaires}

Le domaine de l'éducation est un lieu de débats et de revendications pour les Franco-Ontariens. Depuis la fin du XIX ${ }^{\mathrm{e}}$ siècle, les FrancoOntariens doivent lutter pour obtenir et conserver des droits à des écoles élémentaires et secondaires de langue française. En effet, le développement des écoles franco-ontariennes est régulièrement compromis. L'adoption du tristement célèbre Règlement XVII par le gouvernement ontarien en 1912 représente la plus importante menace au développement des écoles franco-ontariennes. Pendant quinze ans, la crise scolaire provoquée par le Règlement XVII est ponctuée de plusieurs luttes acerbes. Mais cette crise permet néanmoins l'émergence d'une plus grande cohésion et une meilleure convergence des forces en faveur de l'éducation de langue française en Ontario. Créée en 1910, l'Association canadienne-française d'éducation d'Ontario (ACFEO) est la porte-parole incontestée de la communauté franco-ontarienne. Entre 1945 et 1950, les assises de la Commission royale d'enquête sur l'éducation en Ontario (Commission Hope), permet à la communauté franco-ontarienne de constater que ses acquis au niveau de l'enseignement élémentaire et secondaire demeurent précaires. Encore une fois, l'ACFEO se porte à la défense des droits des Franco-Ontariens. Après l'obtention du droit à des écoles secondaires de langue française en 1968, les Franco-Ontariens doivent à nouveau lutter pour obtenir des écoles homogènes de langue française dans de nombreuses localités. Les années 1970 et 1980 sont le théâtre de nombreuses crises scolaires qui ébranlent les rapports entre les francophones et les anglophones en Ontario et au Canada. L'obtention de la pleine gestion des écoles élémentaires et secondaires devient le cheval de bataille des Franco-Ontariens (http://crccf.uottawa.ca/ passeport/IV/IVD.html) 


\section{Annexe 2}

\section{Mission et objectifs}

Reflets s'adresse aux personnes s'intéressant et travaillant à la prévention des problèmes sociaux et de santé, à la promotion et à la défense des droits sociaux, économiques, politiques et culturels des individus, groupes et collectivités, particulièrement les communautés francophones vivant en milieux minoritaires.

Reflets vise à :

- Promouvoir la pensée critique interdisciplinaire sur les politiques, les pratiques institutionnelles et communautaires et sur la formation professionnelle dans les domaines des services sociaux et de la santé, en tenant compte des particularités historico-socio-politiques des francophones en contextes minoritaires;

- Offrir aux intervenantes et aux intervenants un outil de communication permettant de diffuser leurs recherches, de partager des réflexions en lien avec leurs pratiques et l'analyse des politiques sociales, lois et structures organisationnelles et de leurs répercussions sur les populations francophones minoritaires;

- Créer un espace favorisant l'expression et la diffusion de pratiques novatrices ou alternatives dans les secteurs d'intervention touchant les communautés francophones vivant en contextes minoritaires.

\section{Quelques pistes historiques}

Octobre 1992 : Répondant à une invitation de Richard Carrière, une vingtaine de personnes représentant divers milieux de la pratique et diverses régions de la province se réunissent à l'École de service social de l'Université Laurentienne pour étudier les possibilités de publier une revue francophone portant sur l'intervention sociale en Ontario. Yves Vaillancourt, membre fondateur de la revue québécoise Nouvelles pratiques sociales et professeur en service social à l'Université du Québec à Montréal y est invité pour partager son expérience et susciter une réflexion sur les enjeux entourant une telle initiative. 
Février 1993 : Subventionné par le Comité francophone de l'Association des travailleuses et des travailleurs sociaux professionnels de l'Ontario (ATSPO) et réalisé auprès de 734 intervenantes et intervenants francophones de l'Ontario, un sondage permet d'évaluer l'intérêt suscité par la création de la revue. Avec un taux de $35 \%$ de réponses, $99 \%$ des personnes souhaitent lire la revue, $90 \%$ désirent s'y abonner et $44 \%$ se disent prêtes à soumettre des articles.

Novembre 1993 : Lors d'une rencontre à l'École de service social de l'Université d'Ottawa, un comité d'implantation est mis sur pied. (http://sciencessociales.uottawa.ca/Reflets/historique-rubriques pages WWW consultées le 5 août 2015. 


\section{Thèmes abordés — Reflets — 1995 à 2015}

\begin{tabular}{|c|c|c|}
\hline $\begin{array}{l}\text { Année, } \\
\text { volume }\end{array}$ & Thème & Direction \\
\hline $\begin{array}{l}1995 \\
\text { Vol. 1, № } 1\end{array}$ & $\begin{array}{l}\text { Des pratiques sociocommunautaires } \\
\text { à notre image }\end{array}$ & $\begin{array}{l}\text { Madeleine Dubois, Nérée St-Amand et Sonia } \\
\text { Cadieux }\end{array}$ \\
\hline $\begin{array}{l}1995 \\
\text { Vol. 1, № } 2\end{array}$ & $\begin{array}{l}\text { La santé communautaire... défis et } \\
\text { espoirs }\end{array}$ & Michèle Kérisit, Madeleine Dubois et Sonia Cadieux \\
\hline $\begin{array}{l}1996 \\
\text { Vol. 2, № } 1\end{array}$ & $\begin{array}{l}\text { Contrer la violence subie par les } \\
\text { femmes et les enfants en milieu } \\
\text { familial }\end{array}$ & $\begin{array}{l}\text { Marie-Luce Garceau, Richard Carrière, Francine } \\
\text { Boudreau, avec l'assistance de Françoise Arbuckle, } \\
\text { Nicole Bellemare et Marc Charron }\end{array}$ \\
\hline $\begin{array}{l}1996 \\
\text { Vol. 2, № } 2\end{array}$ & Vieillir à l'aube de l'an 2000 & $\begin{array}{l}\text { Marie-Luce Garceau, Noël Simard, Nicole Bellemare } \\
\text { et Marc Charron }\end{array}$ \\
\hline $\begin{array}{l}1997 \\
\text { Vol. } 3, \mathrm{~N}^{0} 1\end{array}$ & $\begin{array}{l}\text { Enfance et familles en contexte } \\
\text { d'appauvrissement }\end{array}$ & Madeleine Dubois et Brigitte Malenfant \\
\hline $\begin{array}{l}1997 \\
\text { Vol. 3, № } 2\end{array}$ & $\begin{array}{l}\text { Visibles et partenaires - pratiques } \\
\text { et recherches féministes }\end{array}$ & $\begin{array}{l}\text { Marie-Luce Garceau avec la collaboration de } \\
\text { Thérèse Grandmont et de Sylvie Larocque } \\
\text { et l'assistance de Marc Charron et de Julie } \\
\text { Boissonneault }\end{array}$ \\
\hline $\begin{array}{l}1998 \\
\text { Vol. } 4, \text { NN}^{0} 1\end{array}$ & Intervention en contexte minoritaire & Michèle Kérisit et Gertrude Mianda \\
\hline $\begin{array}{l}1998 \\
\text { Vol. 4, № } 2\end{array}$ & $\begin{array}{l}\text { Personnes vivant avec une } \\
\text { incapacité }\end{array}$ & $\begin{array}{l}\text { Jean-Marc Bélanger, Richard Carrière avec la } \\
\text { collaboration de Stéphane Laberge et l'assistance } \\
\text { de Marc Charron, Myriam Legault, Michel Blais et } \\
\text { Marie-Luce Garceau }\end{array}$ \\
\hline $\begin{array}{l}1999 \\
\text { Vol. } 5, \text { NN}^{0} 1\end{array}$ & $\begin{array}{l}\text { Pratiques et développement } \\
\text { économique communautaire }\end{array}$ & $\begin{array}{l}\text { Rachid Bagaoui, Donald Dennie } \\
\text { Nérée St-Amand avec la collaboration d'Anick } \\
\text { Mineault }\end{array}$ \\
\hline $\begin{array}{l}1999 \\
\text { Vol. } 5, \text { № }^{2} 2\end{array}$ & $\begin{array}{l}\text { La santé des francophones en } \\
\text { Ontario }\end{array}$ & $\begin{array}{l}\text { Louise Picard, Denise Hébert } \\
\text { Richard Carrière avec l'assistance de Colette } \\
\text { Fraser, Marc Charron, Sylvie Larocque et Anita } \\
\text { Pelletier }\end{array}$ \\
\hline
\end{tabular}




\begin{tabular}{|c|c|c|}
\hline $\begin{array}{l}\text { Année, } \\
\text { volume }\end{array}$ & Thème & Direction \\
\hline $\begin{array}{l}2000 \\
\text { Vol. } 6, \mathrm{~N}^{0} 1\end{array}$ & $\begin{array}{l}\text { Approches d'intervention : définir et } \\
\text { renouveler nos pratiques }\end{array}$ & $\begin{array}{l}\text { Madeleine Dubois, Marie-Luce Garceau avec } \\
\text { l'assistance de Marc Charron }\end{array}$ \\
\hline $\begin{array}{l}2000 \\
\text { Vol. } 6, \mathrm{~N}^{0} 2\end{array}$ & Problèmes sociaux & $\begin{array}{l}\text { Nérée St-Amand, Gertrude Mianda avec la } \\
\text { collaboration de Benoit Veilleux }\end{array}$ \\
\hline $\begin{array}{l}2001 \\
\text { Vol. } 7, \mathrm{~N}^{0} 1\end{array}$ & $\begin{array}{l}\text { Santé mentale et les défis de l'an } \\
2001\end{array}$ & $\begin{array}{l}\text { Jean-Marc Bélanger, Michel-André Beauvolsk et } \\
\text { l'assistance de Julie Boissonneault }\end{array}$ \\
\hline $\begin{array}{l}2001 \\
\text { Vol. } 7, \mathrm{~N}^{0} 2\end{array}$ & Le travail social en Ontario & $\begin{array}{l}\text { Richard Carrière, Adje van de Sande et l'assistance } \\
\text { de Marc Charron et Marie-Luce Garceau }\end{array}$ \\
\hline $\begin{array}{l}2002 \\
\text { Vol. } 8, \mathrm{~N}^{0} 1\end{array}$ & $\begin{array}{l}\text { La réadaptation : son visage français } \\
\text { en Ontario }\end{array}$ & Lynn Casimiro et Louis. E. Tremblay \\
\hline $\begin{array}{l}2003 \\
\text { Vol. } 9, N^{0} 1\end{array}$ & $\begin{array}{l}\text { Le genre en contexte : pratiques } \\
\text { sociales et représentations }\end{array}$ & $\begin{array}{l}\text { Christiane Bernier, Cécile Coderre et } \\
\text { Jacinthe Michaud }\end{array}$ \\
\hline $\begin{array}{l}2003 \\
\text { Vol. } 9, \mathrm{~N}^{0} 2\end{array}$ & Travail et mieux-être & Marc Charron et Marie-Luce Garceau \\
\hline $\begin{array}{l}2004 \\
\text { Vol. } 10, N^{0} 1\end{array}$ & Jeunes et intervention sociale & Marc Molgat \\
\hline 2005, Vol. 11 & Exclusion sociale & Madeleine Dubois et Michèle Kérisit \\
\hline 2006, Vol. 12 & Spiritualité et intervention sociale & Nérée St-Amand et Noël Simard \\
\hline $\begin{array}{l}2007 \\
\text { Vol. } 13\end{array}$ & La violence dans tous ses états & $\begin{array}{l}\text { Myriam Bals avec l'assistance de Richard Carrière } \\
\text { et Marie-Luce Garceau }\end{array}$ \\
\hline $\begin{array}{l}2008 \\
\text { Vol. } 14\end{array}$ & Travail, jeunesse et intervention & Lilian Negura \\
\hline $\begin{array}{l}2009 \\
\text { Vol. } 15\end{array}$ & $\begin{array}{l}\text { Paternités : enjeux et perspectives } \\
\text { (Première partie) }\end{array}$ & $\begin{array}{l}\text { Jean-Martin Deslauriers avec la collaboration de } \\
\text { Nérée St-Amand }\end{array}$ \\
\hline $\begin{array}{l}2010 \\
\text { Vol. } 16, \text { NN}^{0} 1\end{array}$ & $\begin{array}{l}\text { Paternités méconnues (Deuxième } \\
\text { partie) }\end{array}$ & Jean-Martin Deslauriers \\
\hline $\begin{array}{l}2010 \\
\text { Vol. } 16, N^{0} 2\end{array}$ & $\begin{array}{l}\text { L'intervention sociale en contextes } \\
\text { minoritaires : penser la complexité } \\
\text { et la multiplicité des processus de } \\
\text { minorisation }\end{array}$ & $\begin{array}{l}\text { Marie Drolet, Stéphanie Garneau et Madeleine } \\
\text { Dubois }\end{array}$ \\
\hline $\begin{array}{l}2011 \\
\text { Vol. } 17, \mathrm{~N}^{0} 1\end{array}$ & $\begin{array}{l}\text { Psychologisation de l'intervention } \\
\text { sociale }\end{array}$ & Nicolas Moreau et Simon Lapierre \\
\hline
\end{tabular}




\begin{tabular}{|c|c|c|}
\hline $\begin{array}{l}\text { Année, } \\
\text { volume }\end{array}$ & Thème & Direction \\
\hline $\begin{array}{l}2011 \\
\text { Vol. 17, № } 2\end{array}$ & $\begin{array}{l}\text { Représentations, pratiques et } \\
\text { expérience dans le champ de } \\
\text { santé : tendre des ponts entre des } \\
\text { disciplines }\end{array}$ & $\begin{array}{l}\text { Marguerite Soulière, Geneviève Saulnier et Marie- } \\
\text { Luce Garceau }\end{array}$ \\
\hline $\begin{array}{l}2012 \\
\text { Vol. } 18, N^{0} 1\end{array}$ & $\begin{array}{l}\text { L'intervention communautaire chez } \\
\text { les francophones minoritaires : des } \\
\text { pratiques à découvrir }\end{array}$ & $\begin{array}{l}\text { Marie-Luce Garceau, Stéphane Richard et } \\
\text { Sébastien Savard avec la collaboration de Marc } \\
\text { Charron }\end{array}$ \\
\hline $\begin{array}{l}2012 \\
\text { Vol. } 18, N^{0} 2\end{array}$ & $\begin{array}{l}\text { Les inégalités sociales de santé chez } \\
\text { les communautés francophones } \\
\text { en situation minoritaire (CFSM) au } \\
\text { Canada }\end{array}$ & $\begin{array}{l}\text { Monique Benoit, Louise Bouchard, Marie-Luce } \\
\text { Garceau et Anne Leis }\end{array}$ \\
\hline $\begin{array}{l}2013 \\
\text { Vol. } 19, \mathrm{~N}^{0} 1\end{array}$ & $\begin{array}{l}\text { Approches structurelles et } \\
\text { intervention sociale }\end{array}$ & $\begin{array}{l}\text { Simon Lapierre, Joscelyne Levesque et Nérée } \\
\text { St-Amand }\end{array}$ \\
\hline $\begin{array}{l}2013 \\
\text { Vol. } 19, \mathrm{~N}^{0} 2\end{array}$ & $\begin{array}{l}\text { La souffrance psychique et } \\
\text { morale au travail : enjeux pour les } \\
\text { professionnels du secteur de la } \\
\text { santé et des services sociaux }\end{array}$ & Stéphane Richard et Melchior Mbonimpa \\
\hline $\begin{array}{l}2014 \\
\text { Vol. 20, No } 1\end{array}$ & $\begin{array}{l}\text { La formation pratique : allier } \\
\text { milieux de pratique et milieux } \\
\text { d'enseignement }\end{array}$ & $\begin{array}{l}\text { Dominique Mercure, Marie-Luce Garceau, } \\
\text { Madeleine Dubois et Jacynthe Mayer }\end{array}$ \\
\hline $\begin{array}{l}2014 \\
\text { Vol. } 20, N^{0} 2\end{array}$ & $\begin{array}{l}\text { L'accès aux services sociaux et de } \\
\text { santé en français et la formation } \\
\text { des professionnels en situation } \\
\text { francophone minoritaire canadienne }\end{array}$ & $\begin{array}{l}\text { Marie Drolet, Claire-Jehanne Dubouloz, Josée } \\
\text { Benoît et Madeleine Dubois }\end{array}$ \\
\hline $\begin{array}{l}2015 \\
\text { Vol. 21, №1 }\end{array}$ & $\begin{array}{l}\text { Violences faites aux femmes et } \\
\text { contextes minoritaires }\end{array}$ & $\begin{array}{l}\text { Isabelle Côté, Simon Lapierre et Joscelyne } \\
\text { Levesque }\end{array}$ \\
\hline
\end{tabular}

